\title{
Cometary spin-down
}

The rotation rate of a comet more than halved in two months - a much greater change than has previously been observed. This suggests that the comet is in a distinct evolutionary state and might soon reorient itself. SEE LETTER P.186

\section{JESSICA AGARWAL}

K ilometre-sized chunks of ice and dust known as cometary nuclei were left over from the formation of the Solar System ${ }^{1}$. The vast majority of these objects orbit the Sun in one of two cometary reservoirs beyond the orbit of Neptune: the Kuiper belt and the Oort cloud. When an object from one of these reservoirs enters the inner Solar System, it becomes an active comet - its ice is transformed into gas and carries along embedded dust to form a diffuse envelope (coma) and tail. On page 186, Bodewits et al. ${ }^{2}$ report a dramatic decrease in the rotation rate of comet 41P/Tuttle-GiacobiniKresák (comet 41P) indicating that this object could soon enter a phase of rotational instability and reorientation that has never before been seen in a comet.

A rotating celestial body that orbits the Sun without being perturbed has a constant spin state - its rotation rate and the orientation of its axis of rotation relative to inertial space (represented approximately by the positions of stars) are fixed. But, in practice, many factors can change a body's spin state. These include the gravitational pull of other objects, collisions, asymmetric emission of thermal radiation from the body ${ }^{3}$ and, particularly in the case of comets, the recoil force from the asymmetric release of gas.

Gas that streams from a comet's surface accelerates the region of origin in the opposite direction, like a rocket engine (Fig. 1). If the direction of this acceleration does not cross the body's centre of mass, it will produce a turning effect called a torque. And if the time-averaged torques on all surface elements do not cancel each other out, they will alter the comet's spin state. Outgassing forces will also affect the body's orbit around the Sun ${ }^{4}$.

Moderate changes in rotation rate have been observed in several comets - in particular, those visited by spacecraft, for which high-quality data are available. For comet 67P/Churyumov-Gerasimenko, the target of the European Space Agency's Rosetta mission, a clear connection has been established between outgassing-induced torques and changes in rotation rate . $^{5}$

If a comet is spun up to a rotation rate at which the centrifugal force near the equator surpasses gravitational and cohesive forces, landslides and partial or even catastrophic fragmentation can occur ${ }^{6-8}$. Such events would be accompanied by strong sublimation (transformation of ice into gas) and dust production from newly exposed areas, which is one possible cause of sudden increases in brightness called outbursts.

Comet $41 \mathrm{P}$ is a small $(1.4-2.0 \mathrm{~km}$ in diameter) body that originated from the Kuiper belt and was pulled into its current orbit in the inner Solar System by the gravity of Jupiter.
During previous passes by the Sun, known as perihelion passages, the comet had a high level of outgassing activity, given its small size ${ }^{9}$. It passed by Earth at only one-seventh of the Earth-Sun distance (an astronomical unit, AU) on 1 April 2017, and had its closest approach to the Sun at a distance of about $1 \mathrm{AU}$ on 12 April.

Bodewits et al. observed comet 41P in March 2017 using the Discovery Channel Telescope at the Lowell Observatory in Arizona, and then in May using the UltraViolet-Optical Telescope on board the Swift space observatory. Over the two-month interval between their observations, the authors found that the comet's rotation period increased from an already long 20 hours to more than 46 hours. Such a high rate of change has not been seen in a comet before.

The authors conclude that comet $41 \mathrm{P}$ must be subject to an extremely effective torque. They suggest that this feature could be caused by outgassing from a particularly active area far from the body's rotation axis, oriented such that the gas flows in approximately the same direction as the rotation. The efficiency of the torque is enhanced by the comet's comparatively small size, high outgassing rate and slow overall rotation.

Bodewits and colleagues extrapolated the comet's rotation period in time to explore the body's past and future spin states (see Fig. 4 of the paper ${ }^{2}$ ). Assuming comparable torques during past perihelion passages, the authors found that the comet could have been rotating with a period of about 5 hours, which is near the fragmentation limit, before 2006. They hypothesize that this rapid rotation might be linked to a bright outburst that occurred during the comet's 2001 perihelion passage ${ }^{9}$.

For instance, the rotation could have induced a landslide or partial fragmentation in the comet, which would have been visible as an outburst. Alternatively, or in addition, the event behind the outburst might have uncovered an active area that 
is now causing the strong torque. A similar sequence of events could have occurred in comet $103 \mathrm{P} /$ Hartley 2, which was visited by the Deep Impact Extended Investigation (DIXI) space mission ${ }^{8,10}$ in 2010.

Extrapolating comet 41P's rotation rate forward in time, Bodewits et al. predict that the period would have exceeded 100 hours in mid-2017. Such an extremely slow rotation would no longer stabilize the comet's spatial orientation, so that even small torques could make it wobble like a spinning top. If the current strong torque persists, it might eventually drive the comet to spin up again, possibly about a different axis.
A change in comet 41P's rotation axis would affect the seasonal distribution of heating across the body's surface, the associated levels of activity and the pattern of mass transport between different regions ${ }^{11}$. The global process of cometary erosion might therefore be redirected. Observations from the end of the 2017 activity period and from the next perihelion passage in 2022 could document this yetto-be-seen phase of cometary evolution, and reveal valuable information about the nature of comets and other planetary bodies.

Jessica Agarwal is at the Max Planck Institute for Solar System Research,
37077 Göttingen, Germany.

e-mail:agarwal@mps.mpg.de

1. Whipple, F. L. Astrophys. J. 111, 375-394 (1950).

2. Bodewits, D., Farnham, T. L., Kelley, M. S. P. \& Knight, M. M. Nature 553, 186-188 (2018).

3. Rubincam, D. P. Icarus $148,2-11$ (2000)

4. Marsden, B. G. Astron. J. 74, 720-734 (1969)

5. Keller, H. U., Mottola, S., Skorov, Y. \& Jorda, L. Astron. Astrophys. 579, L5 (2015).

6. Hirabayashi, M. et al. Nature 534, 352-355 (2016).

7. Jewitt, D. et al. Astrophys. J. 829, L8 (2016).

8. Steckloff, J. K., Graves, K., Hirabayashi, M., Melosh, H. J. \& Richardson, J. E. Icarus 272, 60-69 (2016).

9. Combi, M. SOHO SWAN Derived Cometary Water Production Rates Collection (NASA, 2017).

10. A'Hearn, M. F. et al. Science 332, 1396-1400 (2011)

11. Keller, H. U. et al. Mon. Not. R. Astron. Soc. 469, S357-S371 (2017).

\section{Neuronal plasticity in nematode worms}

\section{Neuronal activity induces changes in the connectivity of a neuron called DVB in adult male nematode worms. This discovery provides an opportunity to study a fundamental process in this powerful model organism. SEE ARTICLE P.165}

\section{SCOTT W. EMMONS}

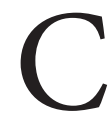
entral to the function of the nervous system is its dynamic ability to undergo changes, for instance in the physiological properties of its constituent neurons, the synaptic connections between them, and the characteristics of individual synapses. The hypothesis that neuronal activity can lead to such plasticity, first proposed by the neurophysiologist Donald Hebb in 1949, is fundamental to brain science, and has been confirmed in many studies ${ }^{1}$. On page 165 , Hart and Hobert ${ }^{2}$ describe an example of experience-dependent neural plasticity in the nematode worm Caenorhabditis elegans, a species in which this phenomenon has been little studied ${ }^{3}$.

It is important to demonstrate this already well-described and widely studied neural phenomenon in a nematode because $C$. elegans is not just any worm, but a powerful experimental model. Genetic studies in C. elegans have led to the discovery of several molecular components common to all nervous systems. Furthermore, a complete map of neural connectivity in the nematode nervous system has been available for more than 30 years ${ }^{4,5}$ - such a connectome is not yet available for any other animal.

Assembly of the C. elegans connectome was made possible not only by the worm's tiny size (1 millimetre long), but also because its cells are constant in number and identity, and its synaptic connections are largely conserved between individuals. These properties, together with the fact that connectivity data were obtained from only a few individuals, have created the impression that the $C$. elegans nervous system is exceptional in having a rigid and constant structure. Intuition suggests that this cannot be the case - the worm's nervous system is so complex that it must be based on dynamic mechanisms. But few examples of variability in C. elegans neurons have been described until now.

The C. elegans inhibitory neuron DVB makes different connections in the worm's two sexes: males and hermaphrodites ${ }^{4,6}$. A single process extends towards the head of the worm in both sexes, and a male-specific outgrowth towards the tail leads to the formation of synaptic connections to a neuron and muscles that control the movement of the male's spicules - a pair of hardened structures that insert into the vulva of the hermaphrodite during mating ${ }^{6}$ (Fig. 1). The formation of these new synapses, and the loss of some old ones, mean that spicule movement comes under the

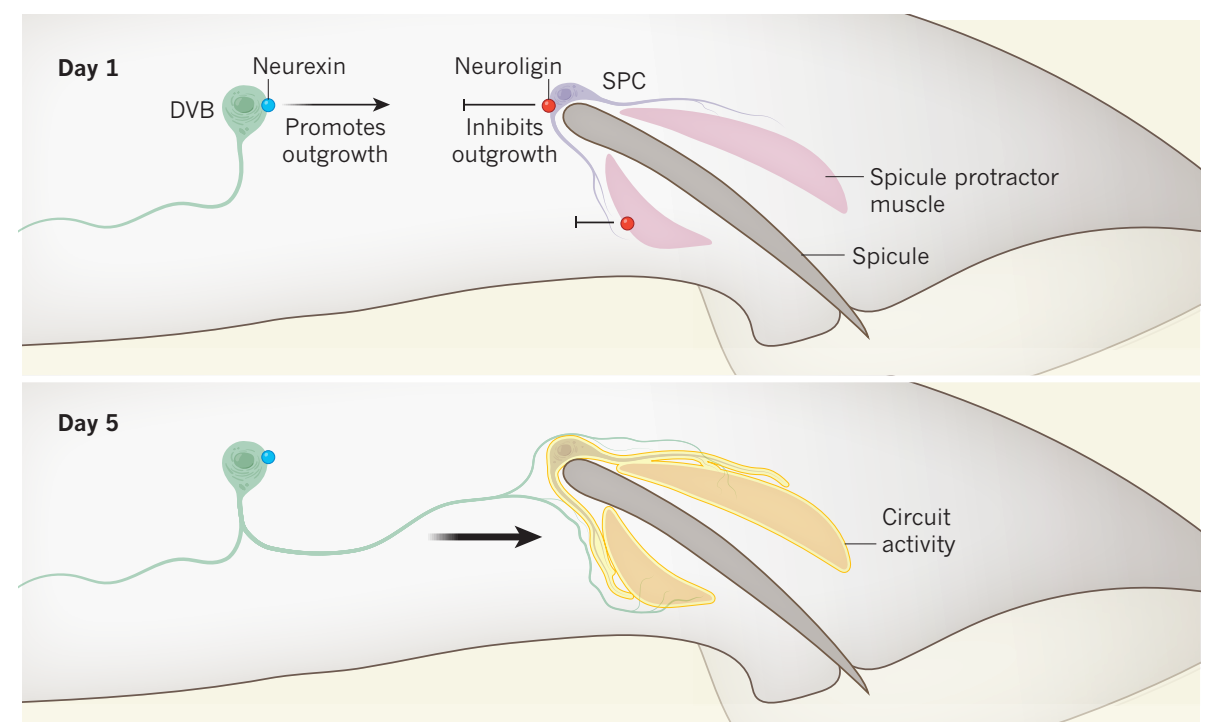

Figure 1 | Activity-dependent neuronal outgrowth in nematodes. Hart and Hobert ${ }^{2}$ examined the neuron DVB in nematode worms (Caenorhabditis elegans). They report that, between days one and five of adulthood in male worms, DVB grows towards, and makes synaptic connections onto, spicule protractor muscles and the spicule neuron SPC, which control a male-specific mating behaviour involving movement of a structure called the spicule. This outgrowth is regulated, at least in part, by two celladhesion proteins: neurexin is expressed by DVB and promotes outgrowth; and neuroligin is expressed by the spicule protractor muscles and SPC, and inhibits outgrowth. The authors show that the expression of neuroligin is repressed when the male undergoes copulatory behaviours, activating these muscles and SPC - DVB outgrowth is therefore activity dependent. 